\title{
AUTOMATIC VEHICLE RECOGNITION FOR URBAN TRAFFIC MANAGEMENT
}

\author{
M. Mohammadi*1, F. Tabib Mahmoudi ${ }^{1}$, M. Hedayatifard ${ }^{2}$ \\ ${ }^{1}$ Dept. of Geomatic Engineering, Civil Engineering Faculty, Shahid Rajaee Teacher Training University, Tehran, Iran - \\ m.mohammadi.ncc@gmail.com, fmahmoudi@sru.ac.ir \\ ${ }^{2}$ Geodesy and Geomatic Engineering Faculty, Khaje Nasir Toosi University, Tehran, Iran - mh_hedayati290@yahoo.com
}

Commission VI, WG VI/4

KEY WORDS: Vehicle Detection, Image pyramid, Segmentation, Object Based Image Analysis, structural features

\begin{abstract}
:
Automatic vehicle recognition has an important role for many applications such as supervision, traffic management and rescue tasks. The ability of online supervision on the distribution of vehicles in urban environments prevents traffic, which in turn reduces air pollution and noise. However, this is extremely challenging due to the small size of vehicles, their different types and orientations, and the visual similarity to some other objects in very high resolution images. In this paper, an automatic vehicle recognition algorithm is proposed based on very high spatial resolution aerial images. In the first step of the proposed method, by generating the image pyramid, the candidate regions of the vehicles are recognized. Then, performing reverse pyramid, decision level fusion of the vehicle candidates and the land use/cover classification results of the original image resolution are performed in order to modify recognized vehicle regions. For evaluating the performance of the proposed method in this study, Ultracam aerial imagery with spatial resolution of $11 \mathrm{~cm}$ and 3 spectral bands have been used. Comparing the obtained vehicle recognition results from the proposed decision fusion algorithm with some manually selected vehicle regions confirm the accuracy of about \%80. Moreover, the $\% 78.87$ and 0.71 are respectively the values for overall accuracy and Kappa coefficient of the obtained land use/cover classification map from decision fusion algorithm.
\end{abstract}

\section{INTRODUCTION}

Considering the speed of construction in large urban areas, traffic management is investigated as one of the most challenging issues in urban management.

Remote sensing images are widely used to monitor vehicles, due to the ability of sensors in providing a complete coverage of the area of interest.

Compared with satellite imagery, aerial imagery is usually more considered for vehicle detection and traffic monitoring purposes due to the higher spatial resolution. However, this is extremely challenging due to the small size of vehicles, their different types and orientations, and the visual similarity to some other objects, such as air conditioning in buildings, trash cans and road signs in high resolution images.

In this paper, an automatic vehicle recognition algorithm is proposed based on aerial imagery. This method uses the advantages of object based image analysis and the image pyramid. Moreover, structural features and neighbourhood relations between various types of objects in urban areas are considered.

\section{LITERATURE REVIEW}

The proposed algorithms for vehicle detection in the literature can be categorized into two main groups: shallow-learningbased methods and deep-learning-based methods.

In shallow-learning-based methods, hand-crafted features are engineered and followed by a classifier or cascade of classifiers (Dalal, \& Triggs, 2005; Maji, et al., 2008; Zheng, et al., 2012). However, shallow-learning-based methods do not result in the desired accuracy in vehicle detection task. They have recently been outperformed by deep learning architectures such as Convolution Neural Network (CNN). On the other hand, deep learning-based techniques have been used for vehicle detection because of their outstanding performance in different domains such as images and sounds. Even though region based CNNs have performed well in natural scene images, their performance in aerial images is limited due to the small size and different orientations of the vehicle, complex background in aerial images, and difficulties in fast detection due to the large size of the aerial images.

Lots of research has been carried out on vehicle detection and counting in aerial images over the past years.

Moranduzzo \& Melgani, (2014) proposed a system for car counting in aerial images captured by UAV. They have reduced search space by selecting the regions where cars might exist using a supervised classifier. Then, they extracted feature points using Scale Invariant Feature Transform (SIFT). Support vector machine (SVM) has been used in order to discriminate between the cars and all other objects.

Chen et al., 2016 proposed a super pixel segmentation method for vehicle detection. Another method has been proposed by $\mathrm{Xu}$ et al., 2016 in which linear SVM classifier and Viola-Jones with HOG features are integrated.

Tuermer et al., 2010 presented a new processing chain to improve vehicle detection. First achievement is limitation of the search space for the detector by applying a fast and simple preprocessing algorithm. Second achievement is generation of a reliable detector. This is done by the use of HOG features (Histogram of Oriented Gradients) and their appliance on two consecutive images. A smart selection of this features and their combination is done by the Real AdaBoost (Adaptive Boosting) algorithm.

A new hybrid vehicle detection scheme which integrates the Viola-Jones (V-J) and linear SVM classifier with HOG feature $(\mathrm{HOG}+\mathrm{SVM})$ methods was proposed for vehicle detection (Xu, et al., 2016). Two most famous object detection schemes are the Viola-Jones (V-J) object detection scheme with Adaboost classifier using Haar-like features (Viola, \& Jones, 2001) and linear support vector machine (SVM) using HOG features (HOG + SVM) (Dalal \& Triggs, 2005). A series of studies have 
demonstrated that these two methods achieved very promising results on vehicle detection (Cao et al., 2011; (Xu, et al., 2015). Most of the works proposed in the deep-learning-based methods use convolution neural network for automatic features extraction. A deep learning approach has been introduced by (Ammour, et al., 2017). In this work, the input image has been segmented into small homogeneous regions. Then, the features in the segmented regions are extracted using pre-trained CNN by a sliding-window approach. Windows are classified using Support Vector Machine (SVM) into car and no-car classes. Finally, post-processing is done such as morphological dilation to smooth the detected regions and fill the holes.

\section{PROPOSED METHOD}

As illustrated in figure 1, the proposed automatic vehicle recognition algorithm in this paper is a decision level fusion algorithm between the initial vehicle candidates and land use/cover classification map in order to modify vehicle recognition results. The initial vehicle candidates are recognized by structural object classification based on image pyramid. The land use/cover classification map is also generated in an object based image analysis procedure.

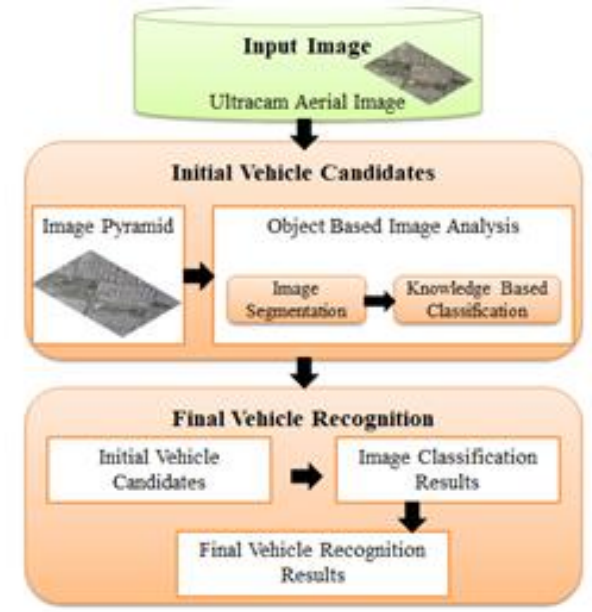

Figure 1. Structure of the proposed vehicle recognition method

\subsection{Initial vehicle candidates}

The proposed algorithm for initial vehicle candidates generation is composed of four main steps;

1) Generating image pyramid by performing Gaussian filter along with down sampling,

2) Performing image segmentation on the pyramid layer, 3) Structural feature measurement on the segmented image objects of pyramid layer. As each segment has special shape, area and structural characteristics, measuring some features such as area, elongation, roundness, compactness and rectangular-fit is so useful for candidate vehicle recognition.

4) Performing knowledge based classification of the image segments into the vehicle and no-vehicle classes in order to produce a binary map containing only the initial candidates of vehicles.

In this research, knowledge classification is performed based on pre-defined thresholds for the structural features in the form of reasoning rules. Some samples of the reasoning rules in this paper are depicted in table 1 .
Table 1. Some sample of reasoning rules for initial vehicle candidate recognition

\begin{tabular}{c|l}
\hline No & \multicolumn{1}{|c}{ Rule } \\
\hline Rule 1 & IF $\mathrm{Th}_{1}<$ area $<=\mathrm{Th}_{2}$ THEN region is Vehicle \\
Rule 2 & IF region== Vehicle AND Rectangular fit $<\mathrm{Th}_{3}$ THEN \\
& $\begin{array}{l}\text { region is No- Vehicle } \\
\text { Rule 3 }\end{array}$ \\
& $\begin{array}{l}\text { IF region== Vehicle AND Elongation }>=\mathrm{Th}_{4} \text { THEN region } \\
\text { is No- Vehicle }\end{array}$ \\
\hline
\end{tabular}

\subsection{Land Use/Cover Classification}

In the first step of the proposed land use/cover classification algorithm in this paper, image segmentation is performed on the original image resolution. Then, by measuring spectral, textural and structural features for each segmented region and determining thresholds based on training samples, classification rules are generated.

Normalized difference vegetation index (NDVI) is measured as spectral feature for vegetation recognition. Moreover, homogeneity in GLCM space is measured for each of the segmented regions as a textural feature.

In this paper, length and elongation are measured as the structural features for road recognition. Roads have long lengths and narrow widths. Therefore, roads are expected to have small amount of elongations.

The proposed classification is a hierarchical process composed of two major categories of rules. The first part is separating vegetation from other objects based on spectral feature. The second part of the classification process is detecting roads, buildings and shadows from non-vegetation objects.

\subsection{Decision Fusion}

In the final step of the proposed automatic vehicle recognition in this paper, a decision level fusion algorithm is performed between initial vehicle candidates and the generated land use/cover classification map. In this procedure, the recognized initial vehicle candidates from pyramid layer should be transferred to the original image resolution by performing inverse pyramid transformation.

Then, considering the meaningful neighbouring relationships between vehicles and other defined object classes, the final and modified vehicle regions are recognized. As four object classes are defined in the land use/cover classification map are buildings, roads, vegetation and shadow, the main neighbouring relationship are in the form of following rules;

- Vehicle candidate regions those are overlaid with road regions or shadow are true vehicles.

- Vehicle candidates those are overlaid with building or vegetation regions are not true vehicles.

- Building, road, shadow and vegetation regions those are overlaid with no-vehicle regions won't change.

\section{RESULTS}

The ability of the proposed method in this study is evaluated based on Ultracam aerial imagery with spatial resolution of 11 $\mathrm{cm}$ and 3 spectral bands (Figure 2).

As it is mentioned in previous section, the initial vehicle candidates should be recognized in the first step of the proposed method. The Gaussian filtering followed by down sampling generates one layer of the image pyramid. Then, multi resolution segmentation algorithm is performed on the pyramid 
layer with 50, 0.1 and 0.5 for scale, shape and compactness parameters, respectively. Figure 3a) illustrates the initial vehicle candidates those were recognized based on structural reasoning rules for each of the pyramid segmented regions.

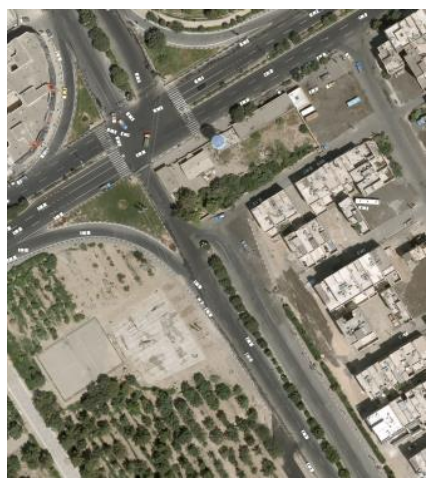

Figure 2. Ultracam aerial imagery

For generating land use/cover classification map containing four object classes; building, road, vegetation and shadow; the multi resolution segmentation algorithm is also performed on the original image resolution with $90,0.5$ and 0.1 for scale, shape and compactness parameters, respectively. Figure $3 \mathrm{~b}$ ) illustrates the obtained results of performing knowledge based classification on the segmented regions.

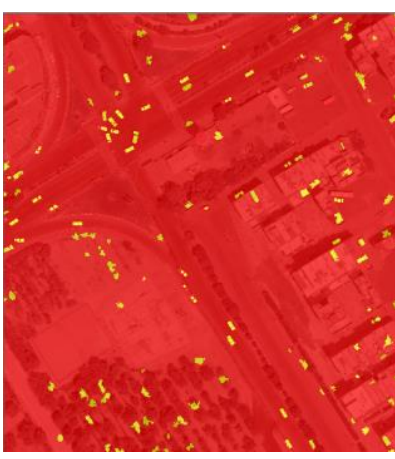

a

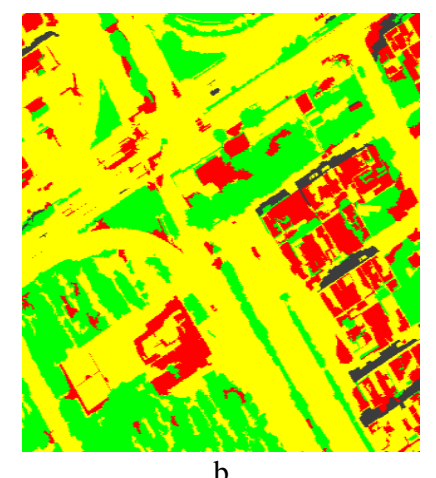

Figure 3. a) Vehicle candidates based on image pyramid, b) object based classification result of the original image

Performing decision level fusion between initial vehicle candidates and all of the object classes in land use/cover classification map generates a final result containing vehicle regions in addition to roads, buildings, vegetation and shadows. Figure 4 illustrates the final vehicle recognition result obtained from the proposed method in this research.
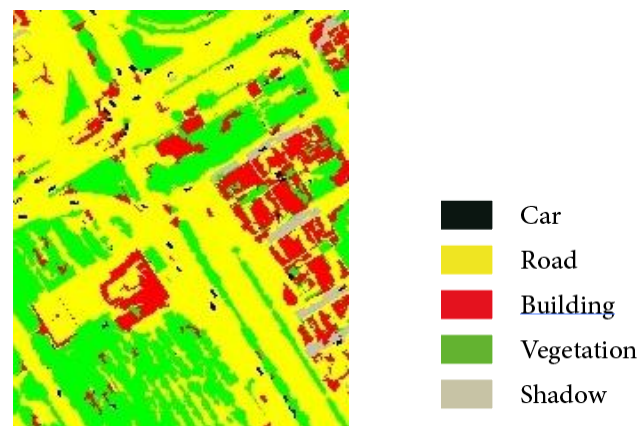

Figure 4. final vehicle recognition result
For the evaluation of the obtained results from the proposed automatic vehicle recognition method, two strategies are used;

1) Accuracy assessment of the only recognized vehicles; in this strategy, some areas containing almost 60000 pixels of the vehicles were selected from image by an expert operator as the ground truth. Then, by comparing these vehicle regions with the automatic recognized vehicles, the accuracy of about $\% 80$ is obtained.

2) Accuracy assessment of the final generated classification map from decision fusion with five object classes, vehicles, buildings, roads, vegetation and shadows; For this evaluation, some regions of interests (ROIs) for each object class were selected by an expert operator in order to generate a ground truth map. The selected ROIs contains about 6000 pixels for each of the buildings and roads classes, 5000 pixels for vegetation, 3000 pixels for shadow and 22000 pixels for vehicles. The $\% 78.87$ and 0.71 are respectively the values for overall accuracy and Kappa coefficient of the obtained land use/cover classification map from decision fusion algorithm.

\section{CONCLUSION}

Automatic vehicle recognition is an important task in many traffic management applications. In this paper, image pyramid is used for generating vehicle candidate regions based on object based image analysis procedure. On the other hand, spectral, textural and structural features of the segmented regions are used for classifying the original image resolution into the land use/cover classification map. Decision level fusion of the initial candidate vehicles and original classification map led to recognized modified vehicle regions. The accuracy of the obtained results from the proposed vehicle recognition strategy in this paper is $80 \%$. Moreover, the final land use/cover classification map has $\% 78.87$ and 0.71 values for overall accuracy and Kappa coefficient, respectively.

\section{REFERENCES}

Ammour, N., Alhichri, H., Bazi, Y., Benjdira, B., Alajlan, N., \& Zuair, M. (2017). Deep learning approach for car detection in UAV imagery. Remote Sensing, 9(4), 312.

Cao, X., Wu, C., Yan, P., \& Li, X. (2011). Linear SVM classification using boosting HOG features for vehicle detection in low-altitude airborne videos. In 2011 18th IEEE International Conference on Image Processing (pp. 2421-2424). IEEE.

Chen, Z., Wang, C., Wen, C., Teng, X., Chen, Y., Guan, H., ... \& Li, J. (2016). Vehicle detection in high-resolution aerial images via sparse representation and superpixels. IEEE Transactions on Geoscience and Remote Sensing, 54(1), 103116.

Dalal, N., \& Triggs, B. (2005). Histograms of oriented gradients for human detection. In international Conference on computer vision \& Pattern Recognition (CVPR'05) (Vol. 1, pp. 886-893). IEEE Computer Society.

Grabner, H., Nguyen, T. T., Gruber, B., \& Bischof, H. (2008). On-line boosting-based car detection from aerial images. ISPRS Journal of Photogrammetry and Remote Sensing, 63(3), 382396. 
Jin, X., \& Davis, C. H. (2007). Vehicle detection from highresolution satellite imagery using morphological shared-weight neural networks. Image and Vision Computing, 25(9), 14221431.

Kembhavi, A., Harwood, D., \& Davis, L. S. (2011). Vehicle detection using partial least squares. IEEE Transactions on Pattern Analysis and Machine Intelligence, 33(6), 1250-1265.

Leitloff, J., Hinz, S., \& Stilla, U. (2010). Vehicle detection in very high resolution satellite images of city areas. IEEE transactions on Geoscience and remote sensing, 48(7), 27952806.

Liu, K., \& Mattyus, G. (2015). Fast multiclass vehicle detection on aerial images. IEEE Geoscience and Remote Sensing Letters, 12(9), 1938-1942.

Liu, W., Yamazaki, F., \& Vu, T. T. (2011). Automated vehicle extraction and speed determination from QuickBird satellite images. IEEE Journal of selected topics in applied earth observations and remote sensing, 4(1), 75-82.

Maji, S., Berg, A. C., \& Malik, J. (2008). Classification using intersection kernel support vector machines is efficient. In 2008 IEEE conference on computer vision and pattern recognition (pp. 1-8). IEEE.

Moranduzzo, T., \& Melgani, F. (2014). Detecting cars in UAV images with a catalog-based approach. IEEE Transactions on Geoscience and remote sensing, 52(10), 6356-6367.

Moranduzzo, T., \& Melgani, F. (2014). Automatic car counting method for unmanned aerial vehicle images. IEEE Transactions on Geoscience and Remote Sensing, 52(3), 1635-1647.

Qu, T., Zhang, Q., \& Sun, S. (2017). Vehicle detection from high-resolution aerial images using spatial pyramid poolingbased deep convolutional neural networks. Multimedia Tools and Applications, 76(20), 21651-21663.

Viola, P., \& Jones, M. (2001). Rapid object detection using a boosted cascade of simple features. CVPR (1), 1, 511-518.

Ruskoné, R., Guigues, L., Airault, S., \& Jamet, O. (1996, August). Vehicle detection on aerial images: A structural approach. In Proceedings of 13th International Conference on Pattern Recognition (Vol. 3, pp. 900-904). IEEE.

Salehi, B., Zhang, Y., \& Zhong, M. (2012). Automatic moving vehicles information extraction from single-pass WorldView-2 imagery. IEEE Journal of selected topics in applied earth observations and remote sensing, 5(1), 135-145.

Shao, W., Yang, W., Liu, G., \& Liu, J. (2012, July). Car detection from high-resolution aerial imagery using multiple features. In 2012 IEEE International Geoscience and Remote Sensing Symposium (pp. 4379-4382). IEEE.

Tang, T., Zhou, S., Deng, Z., Zou, H., \& Lei, L. (2017). Vehicle detection in aerial images based on region convolutional neural networks and hard negative example mining. Sensors, 17(2), 336.

Tayara, H., Soo, K. G., \& Chong, K. T. (2018). Vehicle detection and counting in high-resolution aerial images using convolutional regression neural network. IEEE Access, 6, 22202230 .

Tuermer, S., Leitloff, J., Reinartz, P., \& Stilla, U. (2010). Automatic vehicle detection in aerial image sequences of urban areas using $3 \mathrm{~d}$ hog features. Photogrammetric Computer Vision and Image Analysis, 28(Part 3B), 50-54.

Xu, Y., Yu, G., Wang, Y., \& Wu, X. (2015). Vehicle detection and tracking from airborne images. In 15th COTA International Conference of Transportation ProfessionalsChinese Overseas Transportation Association (COTA) Beijing Jiaotong UniversityTransportation Research BoardInstitute of Transportation Engineers (ITE) American Society of Civil Engineers.

Xu, Y., Yu, G., Wang, Y., Wu, X., \& Ma, Y. (2016). A hybrid vehicle detection method based on viola-jones and HOG+ SVM from UAV images. Sensors, 16(8), 1325.

Zheng, Z., Wang, X., Zhou, G., \& Jiang, L. (2012). Vehicle detection based on morphology from highway aerial images. In 2012 IEEE International Geoscience and Remote Sensing Symposium (pp. 5997-6000). IEEE.

Zheng, Z., Zhou, G., Wang, Y., Liu, Y., Li, X., Wang, X., \& Jiang, L. (2013). A novel vehicle detection method with high resolution highway aerial image. IEEE Journal of Selected Topics in Applied Earth Observations and Remote Sensing, 6(6), 2338-2343. 